\title{
红外预警卫星系统虚警抑制技术综述
}

\author{
李文杰 ${ }^{1}$, 间世强 ${ }^{1}$, 胡 否 $^{2}$, 吴亚宏 ${ }^{1}$, 王成良 ${ }^{1}$, 欧阳琰 ${ }^{1}$
}

(1. 空军预警学院, 湖北 武汉 430019; 2. 解放军 95806 部队, 北京 100076)

\begin{abstract}
摘要: 红外预警卫星系统作为先进的红外探测装备, 是反导预警体系中的重要组成部分, 但是红外 预警卫星系统的虚警问题会严重影响系统作战效能的发挥。在概述红外预警卫星虚警的定义和表现 形式的基础上，分析了虚警的产生原因与分类，最后探讨了红外预警卫星系统虚警抑制的相关技术 和研究方向, 为相关领域的研究提供参考信息。
\end{abstract}

关键词：红外预警卫星；反导预警；虚警；作战效能

中图分类号：TP732.2 文献标识码：A 文章编号：1001-8891(2020)-02-0115-06

\section{A Review of False Alarm Suppression Technology for Infrared Early Warning Satellite System}

\author{
LI Wenjie ${ }^{1}$, YAN Shiqiang ${ }^{1}$, HU Lei ${ }^{2}$, WU Yahong ${ }^{1}$, WANG Chengliang ${ }^{1}$, OUYANG Yan ${ }^{1}$ \\ (1. Air Force Early Warning Academy, Wuhan 430019, China; 2. Unit 95806 of People's Liberation Army, Beijing 100076, China)
}

\begin{abstract}
As an advanced infrared detection equipment, the infrared early warning satellite (IEWS) system is an important part of the anti-missile early warning system. However, the false alarm problem of the IEWS system seriously affects the operational effectiveness of the overall system. Based on the definition and manifestation of the IEWS's false alarm problems, the causes and classifications of these problems are analyzed. Related technologies and research directions for false alarm suppression of the IEWS system are also discussed. This research will serve as a reference for future work in related fields.
\end{abstract}

Key words: infrared early warning satellite, anti-missile early warning, false alarm, operational effectiveness

\section{0 引言}

红外预警卫星是一种先进的星载红外探测系统, 其功能是探测和跟踪弹道导弹飞行时的高温尾焰红 外辐射, 判明敌方弹道导弹发射地点和飞行方向并进 行告警, 是反导预警体系中的重要组成部分。同地面 导弹预警雷达相比较, 具有监视区域大、预警时间长、 反应灵敏、不易受地形干扰等优点。

美国和俄罗斯发展红外预警卫星系统已有数十 年，在实际作战值班的过程中发现存在虚警问题。虚 警是对欺骗或错误事件的报告, 会导致不必要的作战 资源浪费, 此外, 虚警问题还会导致值班人员降低警 惕性, 影响决策者的判断, 从而严重影响反导预警的 整体作战效能。因此, 研究红外预警卫星系统的虚警 问题与其抑制技术的必要性十分迫切。
针对上述问题, 本文从红外预警卫星系统虚警问 题的表现形式出发, 分析虚警的产生原因与分类, 最 后探讨了当前虚警抑制的关键技术和主要研究方向。

\section{1 虚警问题概述}

虚警的定义为 ${ }^{[1]}$ : “电子系统接收机在无目标信号 的情况下, 检测出有信号的指示, 这是由于系统内部 噪声、外部噪声或干扰超过门限所致, 这种错误称为 虚警”。由此可见, 虚警是错误的告警事件, 即当没 有目标时系统却对非目标事件进行告警。

历史上曾出现过多次红外预警卫星系统的虚警 事件, 1979 年 11 月 9 日, 美国北美防空司令部夏延 山基站、五角大楼的国家军事指挥中心、马里兰州里 奇堡的国家预备军事指挥中心同时收到导弹来袭告 警 ${ }^{[2]}$, 后经查证原始数据确定该事件为虚警, 原因是 
由于技术人员错误地在预警系统中运行反导作战想 定程序，模拟苏联对美国本土的导弹袭击。1980 年 6 月 3 日, 美国预警系统发出苏联发射核导弹的来袭告 警, 预警系统显示的来袭导弹数量成随机变化, 后经 查证该事件原因是由于计算机芯片故障导致。1983 年 9 月 26 日, 苏联刚启用的红外预警卫星系统发出了一 次导弹来袭虚警。当时由于太阳和美国导弹基地相对 于苏联红外预警卫星位于同一方向, 阳光在高空云层 中的强烈反射引发系统告警, 被误判为来自美国的导 弹袭击 ${ }^{[3]}$ 。1990 年 12 月 2 日, 在伊拉克发射 “飞毛 腿” 导弹前数小时, 美国国防支援计划 DSP 卫星发出 导弹来袭警告, 经查证告警目标为一架飞行中的 B-52 轰炸机 ${ }^{[4]}$ 。2014 年全年, 由美国空军太空司令部第 460 部队负责管理运行的天基红外预警卫星系统（space based infrared system, SBIRS）共发生 8000 多次红外 事件，其中仅有 403 次为导弹事件。

上述事件表明造成红外预警卫星系统虚警的原 因多样, 总体上可分为软硬件故障、环境辐射干扰两 类。同时，虚警会导致作战人员警惕性的降低，也会 影响决策者的判断, 甚至进行错误地反击而引发严重 后果, 因此对红外预警卫星的虚警问题进行深入研究 和分析是十分必要的。

\section{2 虚警原因分析}

在红外探测系统中, 虚警率 (false alarm rate, FAR) 是检验装备是否具有实战价值的一项重要指标, 用来表示虚警事件发生的频率。虚警率的定义为 “单 位时间内系统发生虚警事件的平均次数”, 其数学模 型可表示为:

$$
\mathrm{FAR}=\frac{N_{\mathrm{FA}}}{T} \times 100 \%
$$

式中: $N_{\mathrm{FA}}$ 为发生虚警事件的次数; $T$ 为系统的工作 总时间。

由此可见, 降低系统虚警率需要减少单位时间内 发生的虚警事件数目, 而分析虚警事件的表现形式和 产生机理是预防和降低系统虚警问题的前提和基础。 经分析, 虚警事件产生的因素可分为环境因素和系统 因素, 具体内容如图 1 所示。

\section{1 环境因素}

环境因素造成的虚警问题主要来自外部环境中 的虚警源干扰, 使得接受到的热辐射超过了红外探测 系统的告警阈值, 从而引发虚警。根据虚警源方位的 不同, 分为空间虚警源、空中虚警源和地表虚警源。

1）空间虚警源

空间虚警源主要是太空中的星体 (如月球) 以及
航天器反射的太阳光 ${ }^{[5]}$, 其中包含了红外预警卫星能 够响应的探测波段, 而且空间虚警源相对红外预警卫 星的运动特性与弹道导弹相似, 因此在空间虚警源进 入红外预警卫星探测器视野时可能会引发告警。

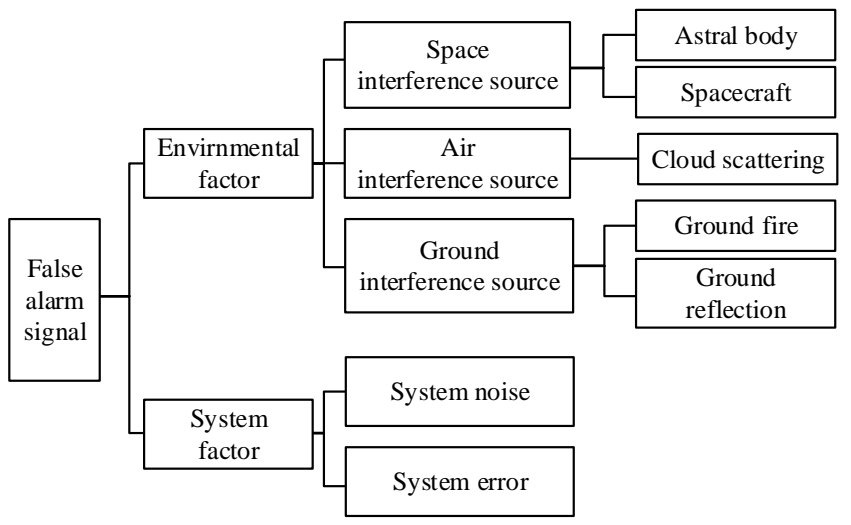

图 1 虚警信号的产生因素

Fig.1 Causes of false alarm signals

\section{2）空中虚警源}

空中虚警源主要是高空卷云反射的太阳光, 高空 卷云由高空分布稀疏的细小冰晶组成, 具有强烈的散 射特性 ${ }^{[6]}$ 。当太阳光朝红外预警卫星方向集中反射时, 探测器将接收到强烈的红外辐射, 引发系统告警。此 外, 高分辨率的红外预警卫星探测器也有可能捕获空 中战斗机尾喷管的热辐射, 从而造成虚警。

\section{3）地表虚警源}

地表虚警源主要是地表高温点和地表强反射区 域。地表高温点为地表燃烧（如森林燃烧）和其他热 辐射源 (如工厂热排放), 其强烈的红外辐射信号与 周围环境形成鲜明对比; 地表强反射区域主要是通过 反射太阳光进入探测器视野形成耀斑而引发虚警, 常 见的地强高反射区域有冰雪覆盖的土地, 沙漠地区以 及平稳的海面 ${ }^{[7]}$ 等。

\section{2 系统因素}

造成虚警的系统因素主要是系统噪声和系统故 障。系统噪声是探测信号中的随机成份, 是系统中固 有的、不可消除的, 会造红外探测图像亮度分别随机 起伏, 若无有效地进行滤除, 可能会引发虚警; 系统 故障是由于系统软件或硬件非正常工作导致的异常 行为。

\section{1) 系统噪声}

系统噪声的组成如图 2 所示, 主要分为热噪声、 电路噪声和探测器噪声。

热噪声是任何绝对零度温度以上的导体和半导 体内部电子均发生的热震动, 其强弱主要受系统内部 的温度变化影响, 而太阳辐射作用和电路热辐射作用 会导致系统内部温度变化。 
电路噪声发生在信息传输的每个环节, 主要包括 线缆噪声、调理电路、电源及偏压噪声和数字模拟转 换 (analog-to-digital converter, ADC) 模块噪声 ${ }^{[8]}$, 理想条件下电路噪声通常保持在一定水平, 但恶劣的 空间环境会对其稳定性造成影响。

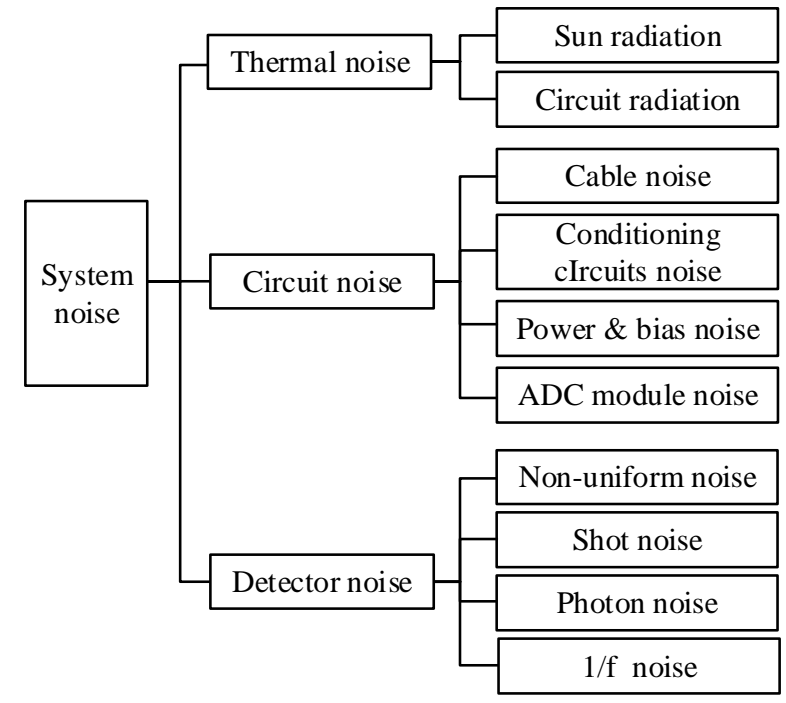

图 2 系统噪声组成

Fig.2 System noise composition

探测器噪声是影响红外系统成像质量的关键因 素, 主要来自两个方面, 一方面来自探测器自身的噪 声, 例如凝视相机探测器中的非均匀性和非线性造成 的噪声等; 另一方面来自扫描相机探测器引入的噪 声。按照噪声的产生机理可以分为散粒噪声、光子噪 声和 $1 / f$ 噪声等。

\section{2) 系统故障}

系统故障可分为软件故障和硬件故障, 是由于系 统自身软、硬件的设计缺陷或外界破坏因素导致的, 使系统处于非正常工作状态, 可能造成信息输入或信 息处理过程中出现不可预料的错误而引发系统告警。

\section{3 虚警抑制相关技术}

通过对虚警事件的表现形式进行描述及虚警的 产生原因进行分析, 为研究红外预警卫星虚警问题的 抑制技术奠定了基础。下文主要从红外成像噪声抑制 技术、红外图像背景抑制技术、红外目标检测与跟踪 算法技术、故障诊断技术以及虚警目标识别技术 5 个 方面展开论述。

\section{1 红外成像噪声抑制技术}

红外成像系统的噪声是影响红外成像质量的主 要因素, 是造成虚警的重要因素, 因此在成像的过程 中就需要对系统进行噪声抑制。根据红外成像噪声的 产生机理可分为动态噪声和非均匀性噪声。

动态噪声以高斯白噪声、椒盐噪声和低频噪声为
主。高斯白噪声随机分布, 在频域具有高斯特征, 高 斯滤波器对其有很好的抑制作用, 其基本原理与均值 滤波器相似, 不同之处是高斯滤波器的模板系数随着 距离模板中心的增大而减小，通过将滤波器窗口内的 像素加权求均值进行输出, 可有效抑制噪声, 平滑图 像。椒盐噪声在红外成像中随机出现且灰度值较为固 定, 中值滤波器通过对滤波器窗口内的图像灰度值进 行排序并取中值作为输出, 可有效消除红外图像中的 椒盐噪声。低频噪声为红外成像系统的 $1 / f$ 噪声, 其 功率谱与频率呈反比, 对红外图像的帧间起伏影响较 大, 可通过小波变换将 $1 / f$ 噪声变为易于清除的白噪 声, 从而满足降噪的目的 ${ }^{[9]}$ 。

非均匀性噪声是由于探测器材料和制造工艺等 因素造成的, 是红外成像系统的固有缺陷, 因此红外 成像系统必须进行非均匀性校正。常用的非均匀性校 正采用基于定标的方法, 主要有一点校正、两点校正、 多点校正以及基于神经网络的改进方法 ${ }^{[10]}$ 等。红外成 像系统的设计阶段就需要进行非均匀性校正, 但在系 统的使用过程中可能会发生温漂效应, 最终影响成像 效果, 因此在轨定标是实现空间红外成像系统准确探 测的基本前提, 目前星上定标 ${ }^{[11]}$ 主要采用太阳、月球、 宇宙冷空间和经校准的黑体源等作为定标校准源。

\section{2 红外图像背景抑制技术}

红外成像系统在对目标进行检测和跟踪前, 背景 抑制是不可缺少的环节, 通过对红外图像进行预处 理, 抑制复杂背景和杂波干扰, 可以提高目标信噪比 以降低虚警率，保证探测的准确性。

传统的红外探测图像背景抑制算法基于单帧图 像, 经典算法有高通滤波算法、中值滤波算法、形态 学滤波算法、罗宾逊滤波算法等 ${ }^{[12]}$, 目前已得到了广 泛的实际应用。上述算法适用于简单背景, 例如海面 和沙漠等单一物理成分构成的区域图像。当背景起伏 较大时, 传统背景抑制方法会有一定残留, 特别当探 测图像中既有简单背景也有复杂背景时, 无法自适应 地抑制不同复杂程度的背景。

针对传统背景抑制算法的不足, 部分学者研究了 复杂背景下的红外弱小目标背景抑制技术，比如基于 背景分类的方法 ${ }^{[13]}$, 基于粒子滤波的方法 ${ }^{[14]}$, 基于空 域滤波的方法 ${ }^{[15]}$, 基于小波变换的方法 ${ }^{[16]}$, 基于复滤 波器组的方法 ${ }^{[17]}$, 基于自适应频域滤波的方法 ${ }^{[18]}$, 基 于光流估计的方法 ${ }^{[19]}$ 等, 均取得较好的背景图像抑制 效果。针对不同应用场景选择适当的算法, 可以有效 提升红外小目标的信噪比, 减少虚警率。

\section{3 红外目标检测与跟踪技术}

红外目标检测与跟踪算法是影响红外探测系统 
虚警率的决定性因素。根据算法检测和跟踪过程的先 后顺序不同，可分为跟踪前检测 (detection before track, DBT) 和检测前跟踪 (track before detection, TBD）两类。

DBT 算法的流程如图 3 所示, 首先对包含目标的 红外图像进行背景抑制, 再通过计算单帧图像的检测 概率和虚警概率确定检测门限, 即可对目标信息进行 分割, 在分割后得到的二值化图像序列中根据目标的 运动特性进行目标轨迹关联, 剔除虚警点, 实现目标 跟踪。经典的 DBT 算法主要有递归最大滤波法 ${ }^{[20]}$ 、 管道滤波法 ${ }^{[21]}$ 和光流法 ${ }^{[22]}$ 等。

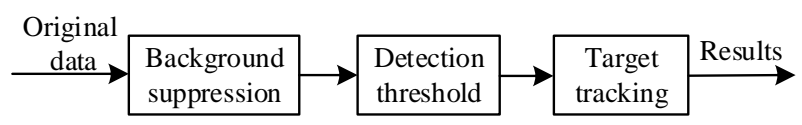

图 3 DBT 算法流程

Fig.3 DBT algorithm flow

TBD 算法的流程如图 4 所示, 首先对包含目标的 红外图像进行背景抑制, 根据目标的运动特性, 跟踪 所有候选目标的运动轨迹, 再根据相邻若干帧图像间 的目标灰度特性或能量变化特性来计算各候选目标 运动轨迹的后验概率, 根据预先设定的阈值, 当满足 间值条件时则认为该目标轨迹是真实的。经典的 DBT 算法主要有基于三维匹配滤波器方法 ${ }^{[23]}$ 、基于 动态规划方法 ${ }^{[24]}$ 、基于高阶相关方法 ${ }^{[25]}$ 、基于粒子 滤波方法 ${ }^{[26]}$ 和基于多级假设检验方法 ${ }^{[27]}$ 等。

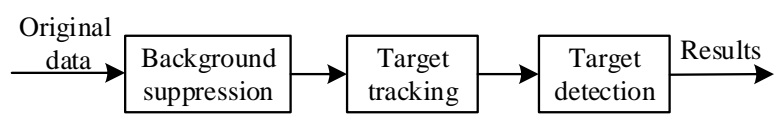

图 4 TBD 算法流程

Fig.4 TBD algorithm flow

DBT 算法和 TBD 算法均是基于多帧信息的红外 小目标检测算法, 区别在于两种算法对于图像序列中 帧间信息的利用顺序有所不同。DBT 算法简单直观， 易于实现, 目前得到了广泛应用, 但是存在抗干扰能 力差, 虚警概率高, 检测概率低的缺点。TBD 算法适 合用来检测图像中低信噪比弱小目标, 相比于 DBT 算法, 相同条件下 TBD 算法的虚警概率更低, 检测 概率更高, 抗干扰能力更强的优点, 但 TBD 算法的 实现较为复杂, 计算量大, 存储量大, 对硬件的性能 要求高, 很难满足实时性要求, 因此应根据具体应用 需求选择合适的检测算法。

\section{4 故障诊断技术}

故障诊断技术的定义 ${ }^{[28]}$ 是: “在不进行设备拆卸 的情况下, 通过相应的方法和技术手段, 在设备运行 过程中掌握其运行状态, 确定是否发生故障并分析出
发生故障的原因, 预报故障未来的发展趋势”。20世 纪 70 年代, 故障诊断技术起源于美国。1971 年, 麻 省理工学院的学者们创新性的提出了运用软件咒余 代替硬件冗余的新思想, 开启了故障诊断技术研究的 开端，此后以美国国家航空航天局（NASA）为代表 的机构对故障诊断技术进行了广泛的应用。此后, 美 国、俄罗斯等国在故障诊断技术方面进行了大量的研 究工作, 经过几十年的发展, 故障诊断技术逐渐成熟, 保证了武器装备的稳定运行。

故障诊断技术大致可以分为 3 类 ${ }^{[29]}$,如图 5 所示, 分别是基于模型的方法、基于信号处理的方法和基于 人工智能 (artificial intelligence, AI) 的方法。

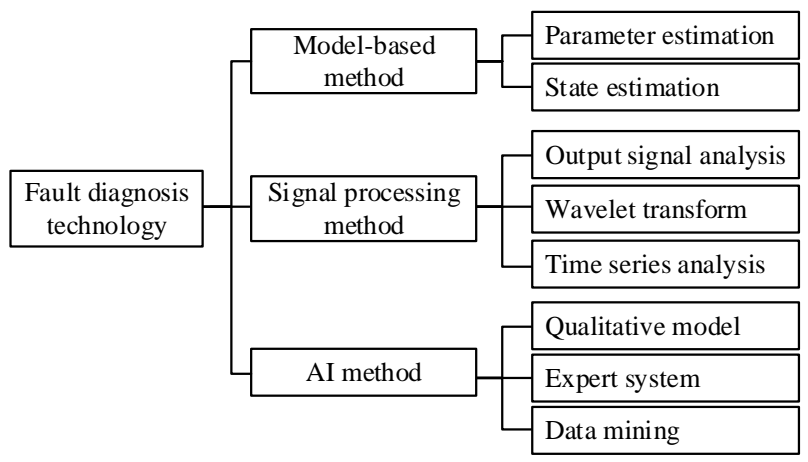

图 5 故障诊断技术分类

Fig.5 Classification of fault diagnosis technology

其中基于人工智能的故障诊断方法已经成为主 要的研究方向。通过积累海量历史数据, 建立故障检 测数据库, 利用定性模型、专家系统、数据挖掘等手 段对卫星与地面站数据集进行分析, 形成系统综合测 试知识库。通过对卫星系统、分系统、设备等参数历 史数据进行分析, 设置故障发生的判别指标, 可对系 统的健康状态进行评估, 并对可能发生的故障做出预 警，从而最大限度地保证了系统运行的可靠性。

\section{5 虚警目标识别技术}

近些年，随着深度学习、图像识别等人工智能技 术的发展以及计算机运算能力的大幅提升, 使得利用 人工智能方法对虚警目标进行识别成为可能。虚警目 标识别的前提是充分了解虚警目标的特性, 可利用事 件记录、反演信息和图像信息等数据, 并研究虚警目 标特征的提取方法。利用全球地表高温点数据集、全 球地表反射率数据集、气象卫星数据集、轨道预报数 据集等资源以形成虚警目标特征库，虚警目标的特征 属性包含虚警目标出现的时间和方位以及虚警目标 的辐射强度和几何信息等。此外, 为避免 “维数灾难” 而影响后期虚警目标识别的效果, 需要对特征数据进 行降维, 图 6 为常用的降维方法的技术分类 ${ }^{[30-31]}$ 。

虚警目标识别的关键在于设计合适的分类算 
法, 实现高准确率的虚警目标识别, 目前常用的分 类算法 ${ }^{[32]}$ 主要有贝叶斯分类算法、支持向量机 （support vector machine, SVM）分类算法、基于关联 规则的分类算法、人工神经网络 (artificial neural networks，ANN）分类算法等。利用虚警目标的特征 库对分类算法进行训练, 并设置合理的评估方法, 使 得模型达到最佳的识别效果。当有红外目标达到系统 告警阈值时, 自动将告警目标的特征信息输入模型进 行属性判别, 若识别为虚警目标, 则进行系统提示以 辅助作战人员进行判别, 并记录虚警事件的特征信 息，不断完善虚警目标识别技术。

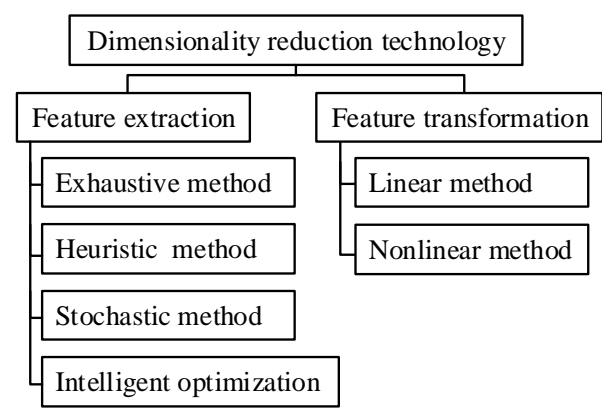

图 6 降维技术分类示意图

Fig.6 Dimension reduction technology classification diagram

\section{4 结语}

本文针对红外预警卫星系统的虚警问题与其抑 制技术展开研究，概述了红外预警卫星虚警问题的表 现形式并进行了分类, 在此基础上对其产生原因进行 分析，最后从红外成像噪声抑制技术、红外图像背景 抑制技术、红外目标检测与跟踪算法技术、故障诊断 技术以及虚警目标识别技术 5 个方面探讨了当前红外 预警卫星系统虚警抑制的相关技术和研究方向，为相 关领域的研究提供了参考信息。

\section{参考文献:}

[1] 杨英科. 信息化作战与电子信息装备试验鉴定术语[M]. 北京：国防 工业出版社, 2011.

YANG Yingke. Informatization Operations and Electronic Information Equipment Test Identification Terminology[M]. Beijing: National Defense Industry Press, 2011.

[2] Angell D J R. NORAD and binational nuclear alert: Consultation and decision making in the integrated command[J]. Defense Analysis, 1988, 4(2): 129-146

[3] Fischer B B. A Cold War Conundrum: The 1983 Soviet War Scare[M]. Washington: Central Intelligence Agency, Center for the Study of Intelligence, 1997.

[4] 杨保平. 导弹预警卫星对抗方式研究[C]//信息产业部电子对抗专业情 报网 2006 年技术交流会, 2006: 20-24.
YANG Baoping. Research on the confrontation mode of missile early warning satellite[C]//Ministry of Information Industry Electronic Countermeasure Professional Information Network Technology Exchange Conference, 2006: 20-24.

[5] 李文杰，宋泽正，李广波，等. 红外预警卫星空间虚警源辐射特性分 析[J]. 红外与激光工程, 2019, 48(3): 61-67.

LI Wenjie, SONG Zezheng, LI Guangbo, et al. Radiation characteristics analysis of space false alarm sources for infrared early warning satellite[J]. Infrared and Laser Engineering, 2019, 48(3): 61-67.

[6] 戴聪明, 赵风美, 刘栋, 等. 强吸收带卷云大气红外辐射特性研究 [J]. 红外与激光工程, 2018, 47(12): 151-157.

DAI Congming, ZHAO Fengmei, LIU Dong, et al. Infrared radiance characteristic of cirrus atmosphere in the strong absorption bands[J]. Infrared and Laser Engineering, 2018, 47(12): 151-157.

[7] 施加宝, 吴振森, 曹运华, 等. 海面中波红外反射率特性研究[J]. 光 子学报, 2009, 38(9): 2372-2375.

SHI Jiabao, WU Zhensen, CAO Yunhua, et al. Study on reflection of sea surface in mid-IR[J]. Acta Photonica Sinica, 2009, 38(9): 2372-2375.

[8] 贾天石, 崔坤, 薛玉龙, 等. 红外探测器测试系统噪声分析与抑制方 法研究[J]. 激光与红外, 2017, 47(11): 1373-1379.

JIA Tianshi, CUI Kun, XUE Yulong, et al. Research on noise analysis and suppression method of infrared detector test system[J]. Laser \& Infrared, 2017, 47(11): 1373-1379.

[9] 朱梦宇, 赵保军, 韩月秋. 用小波变换去除红外图像中 $1 / f$ 噪声的方法 [J]. 北京理工大学学报, 2001, 21(5): 641-644.

ZHU Mengyu, ZHAO Baojun, HAN Yueqiu. A method of removing 1/f noise based on wavelet transform[J]. Journal of Beijing Institute of Technology, 2001, 21(5): 641-644.

[10] 张龙, 董峰, 傅雨田. 基于神经网络的红外图像非均匀性校正 [J]. 红 外技术, 2018, 40(2): 164-169.

ZHANG Long, DONG Feng, FU Yutian. Non-uniformity correction for infrared image using neural networks[J]. Infrared Technology, 2018, 40(2): 164-169.

[11] 陈海龙. 星上定标技术概述[J]. 红外, 2003(6): 9-14.

CHEN Hailong. Overview of on-board calibration techniques[J]. Infrared, 2003(6): 9-14.

[12] 王初阳, 李雪, 梁承玉, 等. 典型的红外图像背景抑制滤波算法研究 [J]. 火力与指挥控制, 2015, 40(8): 33-37.

WANG Chuyang, LI Xue, LIANG Chengyu, et al. Study of algorithms for typical infrared image background suppression filtering[J]. Fire Control \& Command Control, 2015, 40(8): 33-37.

[13] 秦剑, 陈钱, 钱惟贤. 基于背景分类的弱小目标检测算法 [J]. 光电工 程, 2011, 38(1): 23-27.

QIN Jian, CHEN Qian, QIAN Weixian. A detection algorithm for dim and small infrared target based on the classification of the back- 
ground[J]. Opto-Electronic Engineering, 2011, 38(1): 23-27.

[14] 陈东, 林建粦，马德宝. 粒子滤波在空间光电小目标跟踪中的应用 [J]. 光电工程, 2011, 38(3): 1-8.

CHEN Dong, LIN Jianlin, MA Debao. Application of particle filter on the tracking of dim photoelectrical target in space[J]. Opto-Electronic Engineering, 2011, 38(3): 1-8.

[15] 王博, 张建奇. 一种空时域结合滤波的运动弱小目标检测方法[J]. 西 安电子科技大学学报(自然科学版), 2010, 37(3): 524-528.

WANG Bo, ZHANG Jianqi. Moving dim point target detection based on the spatial and temporal combined filter[J]. Journal of Xidian University (Natural Science), 2010, 37(3): 524-528.

[16] 张晓露, 李玲, 辛云宏. 基于小波变换的自适应多模红外小目标检测 [J]. 激光与红外, 2017, 47(5): 647-652.

ZHANG Xiaolu, LI Ling, XIN Yunhong. Adaptive multi-mode infrared small target detection based on wavelet transform[J]. Laser \& Infrared, 2017, 47(5): 647-652.

[17] 秦剑, 陈钱, 钱惟贤. 基于复滤波器组的红外弱小目标检测算法[J]. 强激光与粒子束, 2011, 23(10): 2583-2588.

QIN Jian, CHEN Qian, QIAN Weixian. Dim and small target detection based on complex filter bank[J]. High Power Laser and Particle Beams, 2011, 23(10): 2583-2588.

[18] 张华良, 谢永杰, 张颂, 等. 基于自适应频域滤波的红外弱小目标检 测技术 $[J]$. 应用光学, 2015, 36(4): 630-634.

ZHANG Hualiang, XIE Yongjie, ZHANG Song, et al. Detection of small, low contrast targets based on adaptive frequency filter[J]. Journal of Applied Optics, 2015, 36(4): 630-634.

[19] 秦剑, 陈钱, 钱惟贤. 基于光流估计和自适应背景抑制的弱小目标检 测[J]. 光子学报, 2011, 40(3): 476-482.

QIN Jian, CHEN Qian, QIAN Weixian. A detection algorithm for dim and small infrared target based on the optical flow estimation and the adaptive background suppression[J]. Acta Photonica Sinica, 2011, 40(3): 476-482.

[20] Bronskill J F, Hepburn J S A, Au W K. A knowledge-based approach to the detection, tracking and classification of target formations in infrared image sequences[C]//IEEE Computer Society Conference on Computer Vision \& Pattern Recognition, 1989: 153-158.

[21] 刘靳, 姬红兵. 基于移动式加权管道滤波的红外弱小目标检测 [J]. 西 安电子科技大学学报(自然科学版), 2007, 34(5): 743-747.

LIU Jin, JI Hongbing. Detection method for small targets in the IR image based on the variable weighted pipeline filter[J]. Journal of Xidian University (Natural Science), 2007, 34(5): 743-747.

[22] Shan F, Pridmore T. Image flow field detection[C]//Proceedings of
Third International Conference on Signal Processing, 1996: 1090-1093.

[23] LIU D, ZHANG J, DONG W. Temporal profile based small moving target detection algorithm in infrared image sequences[J]. International Journal of Infrared \& Millimeter Waves, 2007, 28(5): 373-381.

[24] Larson R, Peschon J. A dynamic programming approach to trajectory estimation[J]. IEEE Transactions on Automatic Control, 1966, 11(3): $537-540$.

[25] Liou R J, Azimi-Sadjadi M R. Multiple target detection using modified high order correlations[J]. IEEE Transactions on Aerospace and Electronic Systems, 1998, 34(2): 553-568.

[26] HUA G, HU Y, SHEN Z, et al. Tracking point-source targets in IR noise with neural-network-aided Kalman filter[C]//Signal and Data Processing of Small Targets, 1995, 2561: 589-598.

[27] Blackman S S. Multiple hypothesis tracking for multiple target tracking[J]. IEEE Aerospace and Electronic Systems Magazine, 2004, 19(1): 5-18.

[28] 王剑非. 卫星姿态控制系统的故障诊断研究[D]. 南京: 南京航空航 天大学, 2008.

WANG Jianfei. Research on Fault Diagnosis of Satellite Attitude Control System[D]. Nanjing: Nanjing University of Aeronautics and Astronautics, 2008.

[29] 谢敏, 楼金金, 罗芉. 航天器故障诊断技术综述及发展趋势 [J]. 软件, 2016, 37(7): 70-74.

XIE Min, LOU Xin, LUO Qian. Reviewed and developing trend of spacecraft fault diagnosis technology[J]. Computer engineering \& Software, 2016, 37(7): 70-74.

[30] 吴玲达, 贺玲, 蔡益朝. 高维索引机制中的降维方法综述[J]. 计算机 应用研究, 2006, 23(12): 4-7.

WU Lingda, HE Ling, CAI Yichao. Survey of dimension reduction methods in high-dimensional indexing schemes[J]. Application Research of Computers, 2006, 23(12): 4-7.

[31] 张显东, 霍元铠, 吴乐南, 等. 降维技术与方法综述 $[\mathrm{J}]$. 四川兵工学 报, 2010, 31(10): 1-7.

ZHANG Yudong, HUO Yuankai, WU Lenan, et al. A survey of techniques and methods for dimensionality reduction[J]. Sichuan Ordnance Journal, 2010, 31(10): 1-7.

[32] 何清, 李宁, 罗文娟, 等. 大数据下的机器学习算法综述 [J]. 模式识 别与人工智能, 2014, 27(4): 327-336.

HE Qing, LI Ning, LUO WenJuan, et al. A survey of machine learning algorithms for big data[J]. Pattern Recognition and Artificial Intelligence, 2014, 27(4): 327-336. 